\title{
Spectral plots and the representation and interpretation of biological data
}

\author{
Anirban Banerjee, Jürgen Jost \\ Max Planck Institute for Mathematics in the Sciences, \\ Inselstr.22, 04103 Leipzig, Germany, \\ banerjee@mis.mpg.de, jost@mis.mpg.de
}

October 23, 2018

\begin{abstract}
It is basic question in biology and other fields to identify the characteristic properties that on one hand are shared by structures from a particular realm, like gene regulation, protein-protein interaction or neural networks or foodwebs, and that on the other hand distinguish them from other structures. We introduce and apply a general method, based on the spectrum of the normalized graph Laplacian, that yields representations, the spectral plots, that allow us to find and visualize such properties systematically. We present such visualizations for a wide range of biological networks and compare them with those for networks derived from theoretical schemes. The differences that we find are quite striking and suggest that the search for universal properties of biological networks should be complemented by an understanding of more specific features of biological organization principles at different scales.
\end{abstract}

\section{Introduction}

The large volume of typical data sets produced in modern molecular, cell and neurobiology raises certain systematic questions, or, more precisely, brings new aspects to some old scientific issues. These, or at least the ones we wish to address in this note, are:

1. Given a particular biological structure, which of each features or qualities are universal, that is, shared by other structures within a certain class, and what is unique and special for the structure at hand?

2. Given a large and complex structure, should we focus on particular and specific aspects and quantities in detail, or should we try to obtain, at least 
at some coarse level, a simultaneous representation of all its qualitative features?

Clearly, these questions can be posed, but not answered in such generality. Here, we look at those issues for a particular type of biological data, namely those that are presented as graphs or networks. For these, we introduce and discuss a certain representation, a spectral plot, that allows for analyzing the questions raised above, in both cases with an emphasis on the second alternative.

\section{Graphs and their invariants}

Many biological data sets are, or can be, represented as networks, that is, in terms of the formal structure of a graph. The vertices of a graph stand for the units in question, like genes, proteins, cells, neurons, and an edge between vertices expresses some correlation or interaction between the corresponding units. These edges can be directed, to encode the direction of interaction, for example via a synaptic connection between neurons, and weighted, to express the strength of interaction, like a synaptic weight. Here, for simplicity of presentation, we only consider the simplest type of a graph, the undirected and unweighted one, although our methods apply and our considerations remain valid in the general situation. Thus, an edge expresses the presence of some interaction, connection or direct correlation between two vertices, regardless of its direction or strength. Clearly, this abstraction may loose many important details, but we are concerned here with what it preserves. Thus, we shall investigate the two issues raised in the introduction for, possibly quite large, graphs.

An (unweighted, undirected) graph is given by a set $V$ of vertices $i, j, \ldots$ and a set of edges $E$ which are simply unordered pairs $(i, j)$ of vertices 11 (Usually, one assumes that there can only be edges between different vertices, that is, there are no self-loops from a vertex to itself.) Thus, when the pair $(i, j)$ is in $E$, then the vertices $i$ and $j$ are connected by an edge, and we call them neighbors and write this relation as $i \sim j$. The degree $n_{i}$ of a vertex $i$ is defined to be the number of its neighbors. Already for rather modest numbers of vertices, say 20, the number of different graphs is bewilderingly high ${ }^{2}$ Thus, it becomes impractical, if not impossible, to list all different graphs with a given number of vertices, unless that number is rather small. Also, drawing a graph with a large number of vertices is not helpful for visual analysis because the structure will just look convoluted and complicated instead of transparent. On the other hand, graphs can be qualitatively quite different, and understanding this is obviously crucial for the analysis of the represented biological structures.

\footnotetext{
${ }^{1}$ Some general references for graph theory are 9 14.

${ }^{2}$ As always in mathematics, there is a notion of isomorphism: Graphs $\Gamma_{1}$ and $\Gamma_{2}$ are called isomorphic when there is a one-to-one map $\rho$ between the vertices of $\Gamma_{1}$ and $\Gamma_{2}$ that preserves the neighborhood relationship, that is $i \sim j$ precisely if $\rho(i) \sim \rho(j)$. Isomorphic graphs are considered to be the same because they cannot be distinguished by their properties. In other words, when we speak about different graphs, we mean non-isomorphic ones.
} 
For example, the maximal distance (number of edges) between two vertices in a graph of size $N$ can vary between 1 and $N-1$, depending on the particular graph. When the graph is complete, that is, every vertex is connected with every other one, any two vertices have the distance 1 , whereas for a chain where vertex $i_{1}$ is only connected to $i_{2}, i_{2}$ then also to $i_{3}$, and so on, the first and the last vertex have distance $N-1$. For most graphs, of course, some intermediate value will be realized, and one knows from the theory of random graphs that for a typical graph this maximal distance is of the order $\log N$. So, this maximal distance is one graph invariant, but still, rather different graphs can have the same value of this invariant. Adjoining a long sidechain to a complete subgraph can produce the same value as an everywhere loosely connected, but rather homogeneous graph. The question then emerges whether one should look for other, additional or more comprehensive, invariants, or whether one should adopt an entirely different strategy for capturing the essential properties of some given graph.

In fact, there are many graph invariants that each capture certain important qualitative aspects, and that have been extensively studied in graph theory (see e.g. 9, 14]). These range from rather simple and obvious ones, like maximal or average degree of vertices or distance between them, to ones that reflect more global aspects, like how difficult it is to separate the graph into disjoint components (see e.g. [11]), commmunities (e.g. 24]) or classes, or to synchronize coupled dynamics operating at the individual vertices (e.g. [19]). For the sake of the subsequent discussion, we call these properties cohesion and coherence, resp. Recently, the degree distribution of the vertices received much attention. Here, for each $n \in \mathbb{N}$, one lists the number $k_{n}$ vertices $i$ in the graph with degree $n_{i}=n$ and then looks at the behavior of $k_{n}$ as a function of $n$. In random graphs as introduced by Erdös and Rényi (e.g. 10]), that is, graphs where one starts with a given collection of, say $N$, vertices and to each pair $i, j$ of vertices, one assigns an edge with some probability $0<p<1$, typically $k_{n} \sim e^{-\sigma n}$ for some constant $\sigma$, that is the degree sequence decays exponentially ${ }^{3}$ Barabasi-Albert [8] (and earlier Simon [27]) gave a different random construction where the probability of a vertex $i$ to receive an edge from some other vertex $j$ is not uniform and fixed, but rather depends on how many edges $i$ already has. This was called preferential attachment, that is, the chance of a vertex to receive an additional edge increases when it already possesses many edges. For such a graph, the degree sequence behaves like $k_{n} \sim n^{-\kappa}$ for some exponent $\kappa$, typically between 2 and 3 . Thus, the degree sequence decays like a power law, and the corresponding type of graph is called scale free. They also gave empirical evidence of networks that follow such a power law degree distribution rather than an exponential one. This produced a big fashion, and in its wake, many empirical studies appeared that demonstrated or claimed such a power law behavior for large classes of biological or infrastructural networks. Thus, scalefreeness seemed a more or less universal feature among graphs com-

\footnotetext{
${ }^{3}$ In more precise terms, the degrees are Poisson distributed in the limit of an infinite graph size.
} 
ing from empirical data in a wide range of domains. While this does provide some insights (for some systematic discussion, see e.g. [1, 12, 28]), for example a better understanding of the resilience of such graphs against random deletion of vertices, it also directly brings us to the two issues raised in the introduction:

1. A feature like scalefreeness that is essentially universal in empirical graphs by its very nature fails to identify what is specific for graphs coming from a particular domain. In other words, do there exist systematic structural differents for example between gene regulation, protein-protein interaction or neural networks? Or, asked differently, given an empirical graph, without being told where it comes from, can one identify this domain on the basis of certain unique qualitative features?

2. Graphs with the same degree sequence can be quite different with respect to other qualitative invariants like the coherence, as emphasized in [2, 3. Also, depending on the details of the preferential attachment rule chosen, invariants like the average or maximal distance can vary widely, as observed in 20]. Thus, is there some way to encode many, or even essentially all, important graph invariants simultaneously in some compact manner?

\section{The spectrum of a graph}

In order to provide a positive answer to these question, we shall now introduce and consider the spectrum of a (finite) graph $\Gamma$ with $N$ vertices. For functions $v$ from the vertices of $\Gamma$ to $\mathbb{R}$, we define the Laplacian as

$$
\Delta v(i):=v(i)-\frac{1}{n_{i}} \sum_{j, j \sim i} v(j) .
$$

(Note that in the graph theoretical literature (e.g. 9, 14, 21, 23), it is more customary to put a factor $n_{i}$ in front of the right hand side in the definition of the Laplacian. The choice of convention adopted here is explained in [17, 18. It is equivalent to the one in [11.)

The eigenvalue equation for $\Delta$ is

$$
\Delta u=\lambda u
$$

A nonzero solution $u$ is called an eigenfunction for the eigenvalue $\lambda . \Delta$ then has $N$ eigenvalues, perhaps occurring with multiplicity, that is, not necessarily all distinct. (The multiplicity of the eigenvalue $\lambda$ is the number of linearly independent solutions of (2).) The eigenvalues of $\Delta$ are real and nonnegative (because $\Delta$ is a symmetric, nonnegative operator, see e.g. [17, 18] for details). The smallest eigenvalue is $\lambda=0$. Its multiplicity equals the number of components of $\Gamma$. When $\Gamma$ is connected, that is, has only one component (as we typically assume because one can simply study the different components as graphs in their own 
right), this eigenvalue is simple. The other eigenvalues are positive, and we order the eigenvalues a: 4

$$
\lambda_{1}=0 \leq \lambda_{2} \leq \ldots \leq \lambda_{N}
$$

For the largest eigenvalue, we have

$$
\lambda_{N} \leq 2,
$$

with equality iff the graph is bipartite. Bipartiteness means that the graph consists of two disjoint classes $\Gamma^{\prime}, \Gamma^{\prime \prime}$ with the property that there are no edges between vertices in the same class. Thus, a single eigenvalue determines the global property of bipartiteness.

The eigenvalue $\lambda=1$ also plays a special role as it gives some indication of vertex or motif duplications underlying the evolution of the graph, as systematically explored in [5, 7]. For a biological discussion, see [25, 29, 32. For a general mathematical discussion of the spectrum, see [11, 6] and the references given there.

What we want to emphasize here is that the spectrum constitutes an essentially complete set of graph invariants. At least without the qualification "essentially", this is not literally true, in the following sense: there are examples of nonisomorphic graphs with the same spectrum - such graphs are called isospectral (see [33]). In other words, the spectrum of a graph does not determine the graph completely (see [15] for a graph reconstruction algorithm from the spectrum). Such isospectral graphs, while not necessarily isomorphic, are qualitatively very similar to each other, however. In particular, the spectrum of the graph encodes all the essential qualitative properties of a graph, like cohesion and coherence (e.g. [11, 19, 4). Thus, for practical purposes, this is a good enough set of graph invariants.

In conclusion, the spectrum of a graph yields a set of invariants that on one hand captures what is specific about that graph and on the other hand simultaneously encodes all its important properties.

\section{Visualization of a graph through its spectral plot}

What is more, the spectral plot of a graph is much better amenable to visual inspection than a direct plot of the graph or any other method of representation that we know of. In other words, with a little experience in graph theory, one can quickly detect many important features of a graph through a simple look at its spectral plot. We now display some examples 5

\footnotetext{
${ }^{4}$ Our convention here is different from $19,17,18,6$.

${ }^{5}$ All networks are taken as undirected and unweighted. Thus, we suppress some potentially important aspects of the underlying data, but as our plots will show, we can still detect distinctive qualitative patterns. In fact, one can also compute the spectrum of directed and weighted networks, and doing that on our data will reveal further structures, but this is not explored in the present paper.
} 
First of all, the properties of the visualization will obviously depend on the display style, and this will be described first, see Figure 1. That figure is based on the metabolic network of C.elegans. The first diagram displays the binned eigenvalues, that is, the range $[0,2]$ is divided here into 35 disjoint bins, and the number of eigenvalues that fall within each such bin is displayed, normalized by the total number of eigenvalues (relative frequency plot). The next figure smoothes this out by using overlapping bins, see figure legend for parameter values. The subsequent subfigures instead convolve the eigenvalues with a Gaussian kernel, that is, we plot the function

$$
f(x)=\sum_{\lambda_{j}} \frac{1}{\sqrt{2 \pi \sigma^{2}}} \exp \left(-\frac{\left|x-\lambda_{j}\right|^{2}}{2 \sigma^{2}}\right)
$$

where the $\lambda_{j}$ are the eigenvalues. Smaller values of the variance $\sigma^{2}$ emphasize the finer details whereas larger values bring out the global pattern more conspicuously.

So, this is a network constructed from biological data. We next exhibit spectral plots, henceforth always with the value $\sigma=.03$, of networks constructed by formal schemes that have been suggested to capture important features of biological networks, namely an Erdös-Rényi random network, a Watts-Strogatz small-world network and a Barabási-Albert scale-free network (see Figure 2). It is directly obvious that these spectral plots are very different from the metabolic network. This suggests to us that any such generic network construction misses important features and properties of real biological networks. This will now be made more evident by displaying further examples of biological networks. We shall also see that biological networks from one given class typically have quite similarly looking spectral plots, which, however, are easily disitnguishable from those of networks from a different biological class. First, in Figure 3 , we show some further metabolic networks. Figures 4 and 5 display transcription and protein-protein interaction networks. These still look somewhat similar to the metabolic networks, and this may reflect a common underlying principle. By way of contrast, the neurobiological networks in Figure 6 and the food-webs in Figure 7 are entirely different - which is not at all surprising as they come from different biological scales.

In conclusion, we have presented a simple technique for visualizing the important qualitative aspects of biological networks and for distinguishing networks from

different origins. We expect that our method will further aid formal analysis of biological network data.

\section{References}

[1] R. Albert, A.-L. Barabási, Statistical mechanics of complex networks, Reviews of Modern Physics 74, 2002, 47-97. 
[2] F.M. Atay, T.Bıyıkoğlu, J.Jost, Synchronization of networks with prescribed degree distributions, IEEE Trans. Circuits and Systems I 53 (1), 2006, 92-98.

[3] F.M. Atay, T.Bıyıkoğlu, J.Jost, Network synchronization: Spectral versus statistical properties, Phys.D, to appear

[4] F.M. Atay, J. Jost, A. Wende, Delays, connection topology, and synchronization of coupled chaotic maps, Phys. Rev. Lett. 92 (14), 2004, 144101.

[5] A.Banerjee, J.Jost, Laplacian spectrum and protein-protein interaction networks, e-print: arXiv:0705.3373v1

[6] A.Banerjee, J.Jost, On the spectrum of the normalized graph Laplacian,

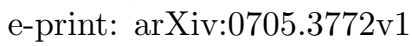

[7] A.Banerjee, J.Jost, Graph spectra as a systematic tool in computational biology, Discrete Appl.Math. to appear, e-print: arXiv:0706.0113v1

[8] A.-L. Barabási, R. A. Albert, Emergence of scaling in random networks, Science 286, 1999, 509-512.

[9] B.Bolobás, Modern graph theory, Springer, 1998

[10] B.Bolobás, Random graphs, Cambridge Univ.Press, ${ }^{2} 2001$

[11] F.Chung, Spectral graph theory, AMS, 1997

[12] S.N. Dorogovtsev, J.F.F. Mendes, Evolution of Networks, Oxford, 2003.

[13] P. Erdős, A. Rényi, On random graphs, Publicationes Mathematicae Debrecen, 6, 1959, 290-297

[14] C.Godsil, G.Royle, Algebraic graph theory, Springer, 2001

[15] M. Ipsen, A. S. Mikhailov, Evolutionary reconstruction of networks, Phys. Rev. E 66(4), 2002

[16] H. Jeong and B. Tombor and R. Albert and Z. N. Oltval and A. L. Barabási, The Large-Scale Organization of Metabolic Networks, Nature, 407(6804), 2000, 651-654

[17] J. Jost, Mathematical methods in biology and neurobiology, monograph, to appear

[18] J. Jost, Dynamical networks, in: J.F.Feng, J.Jost, M.P.Qian (eds.), Networks: from biology to theory, Springer, 2007

[19] J. Jost, M. P. Joy, Spectral properties and synchronization in coupled map lattices, Phys.Rev.E 65(1), 16201-16209, 2001 
[20] J.Jost, Joy, Evolving networks with distance preferences, Phys.Rev.E 66, 36126-36132, 2002

[21] R. Merris, Laplacian matrices of graphs - a survey, Lin. Alg. Appl.198, 1994, 143-176

[22] R. Milo and S. Shen-Orr and S. Itzkovitz and N. Kashtan and D. Chklovskii and U. Alon, Network Motifs: Simple Building Blocks of Complex Networks, Science, 298(5594), 2002, 824-827

[23] B. Mohar, Some applications of Laplace eigenvalues of graphs, in: G.Hahn, G.Sabidussi (eds.), Graph symmetry: Algebraic methods and applications, pp. 227-277, Springer, 1997

[24] M. Newman, The structure and function of complex networks, SIAM Review $45,2003,167-256$

[25] S.Ohno, Evolution by gene duplication, Springer, 1970

[26] S. S. Shen-Orr and R. Milo and S. Mangan and U. Alon, Network Motifs in the Transcriptional Regulation Network of Escherichia Coli, Nature Genetics, 31(1), 2002, 64-68

[27] H.Simon, On a class of skew distribution functions, Biometrika 42, 425440,1955

[28] A.Vázquez, Growing networks with local rules: Preferential attachment, clustering hierarchy and degree correlations, cond-mat/0211528, 2002

[29] A. Wagner, Evolution of gene networks by gene duplications - a mathematical model and its implications on genome organization, Proc. Nat. Acad. Sciences USA 91(10), 1994, 4387-4391

[30] D. J. Watts, S. H. Strogatz, Collective Dynamics of 'Small-World' Networks, Nature, 393(6684), 1998, 440-442

[31] J. G. White and E. Southgate and J. N. Thomson and S. Brenner, The Structure of the Nervous-System of the Nematode CaenorhabditisElegans, Philosophical Transactions of the Royal Society of London Series B-Biological Sciences, 314(1165), 1986, 1-340

[32] K. H. Wolfe, D. C. Shields, Molecular evidence for an ancient duplication of the entire yeast genome, Nature, 387(6634), 1997, 708-713

[33] P.Zhu, R.Wilson, A study of graph spectra for comparing graphs 


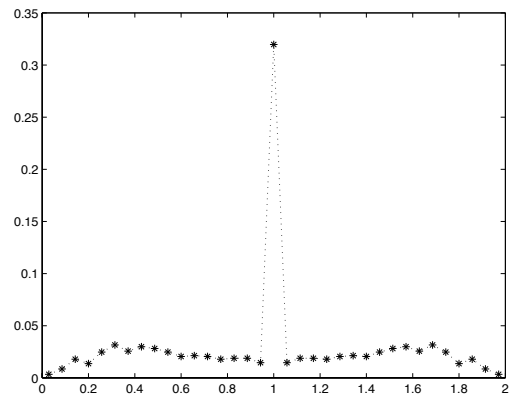

(a)

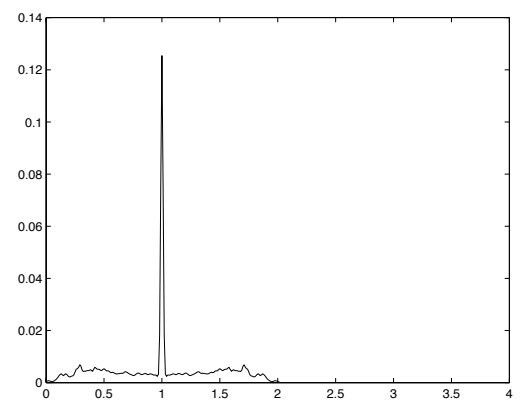

(c)

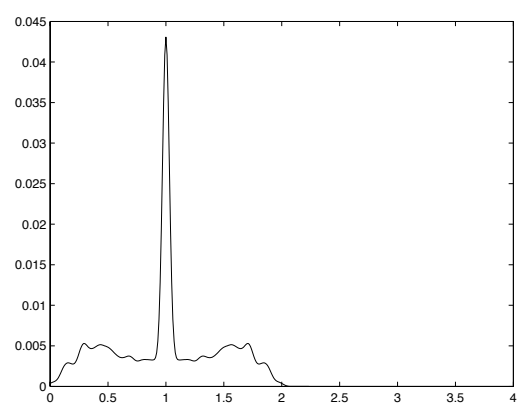

(e)

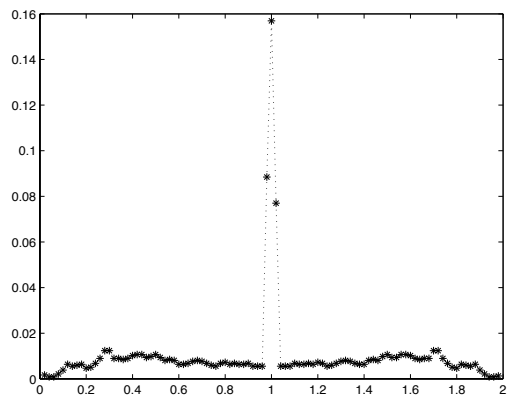

(b)

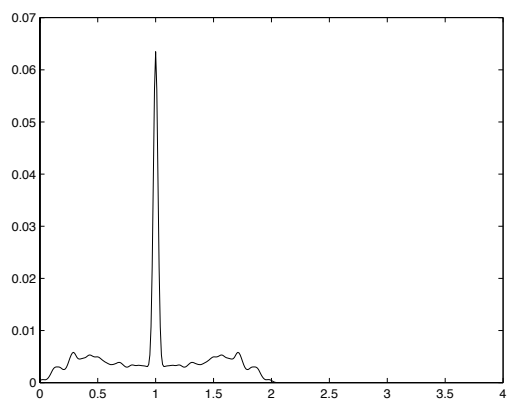

(d)

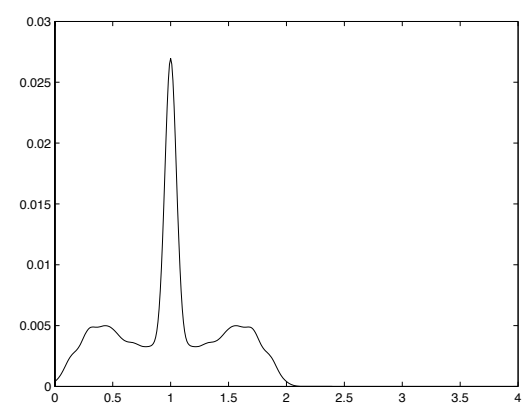

(f)

Figure 1: Spectral plots of metabolic network of Caenorhabditis elegans. Size of the network is 1173. Nodes are substrates, enzymes and intermediate complexes. Data used in [16. Data Source: http://www.nd.edu/ networks/resources.htm/. [Download date: 22nd Nov. 2004]. (a) Relative frequency plot with number of bins $=35$. (b) Relative frequency polygon with overlapping bins with bin width $=0.04$ and number of bins $=99$ and bins are taken as $[0, .04],[.02, .06],[.04, .08], \ldots,[1.96,2]$. (c) with Gaussian kernel with $\sigma=0.01$. (d) with Gaussian kernel with $\sigma=0.02$. (e) with Gaussian kernel with $\sigma=0.03$. (f) with Gaussian kernel with $\sigma=0.05$. 


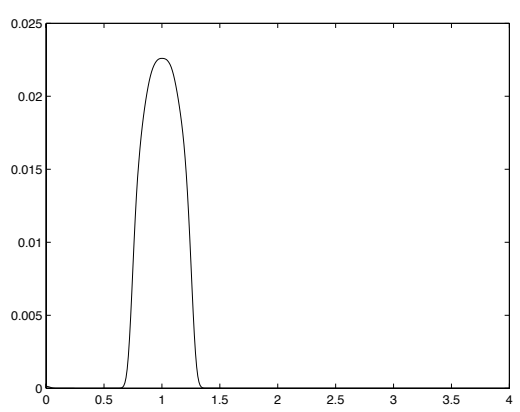

(a)

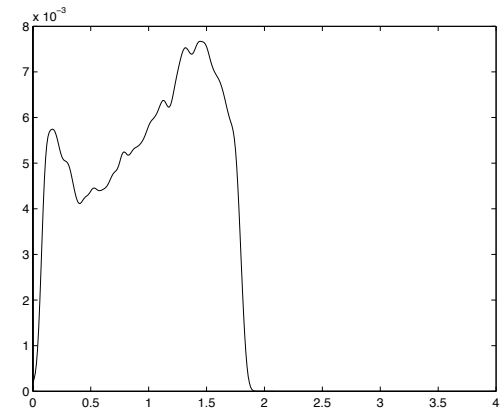

(b)

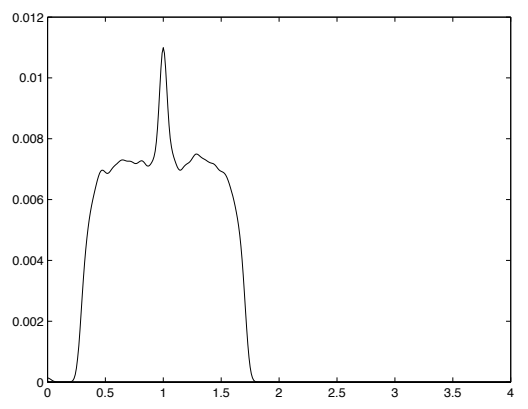

(c)

Figure 2: Specral plots of generic networks. (a) Random network by Erdös and Rényi's model 13 with $p=0.05$. (b) Small-world network by Watts and Stogatz model [30] (rewiring a regular ring lattice of average degree 4 with rewiring probability 0.3). (d) Scale-free network by Albert and Barabási model [8] $\left(m_{0}=5\right.$ and $\left.m=3\right)$. Size of all networks is 1000. All figures are ploted with 100 realization. 


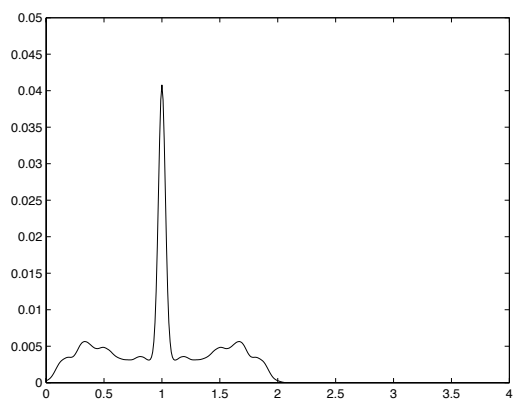

(a)

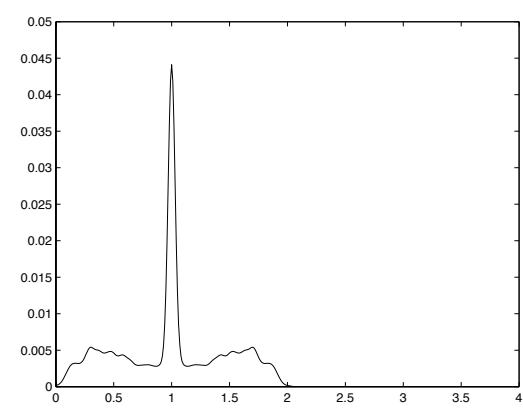

(b)

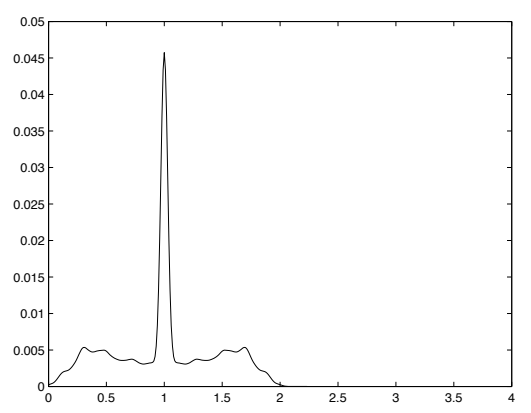

(c)

Figure 3: Metabolic networks. Here nodes are substrates, enzymes and intermediate complexes. Data used in [16. Data Source: http://www.nd.edu/ networks/resources.htm/. [Download date: 22nd Nov. 2004] (a) Pyrococcus furiosus. Network size is 746. (b) Aquifex aeolicus. Network size is 1052. (c) Saccharomyces cerevisiae. Network size is 1511. 


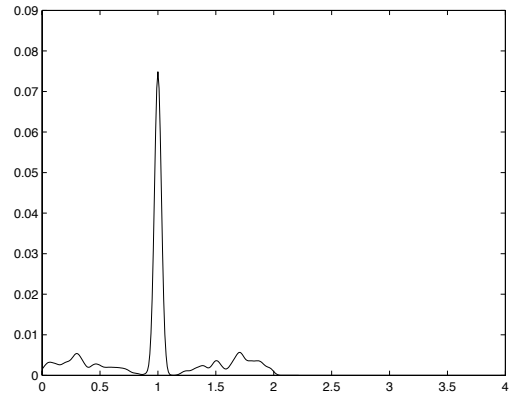

(a)

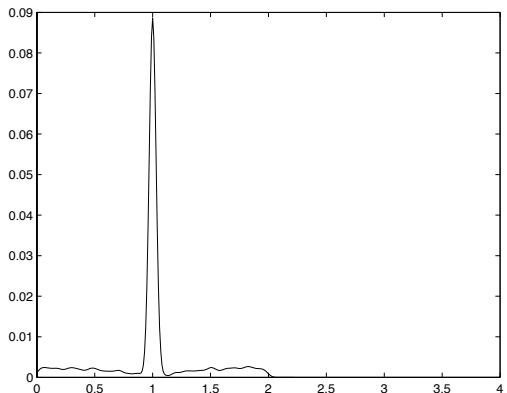

(b)

Figure 4: Transcription networks. Data source: Data published by Uri Alon (http://www.weizmann.ac.il/mcb/UriAlon/ ). [Download date: 13th Oct. 2004]. Data used in [22, 26]. (a) Escherichia coli. Size of the network is 328. (b) Saccharomyces cerevisiae. Size of the network is 662 . 


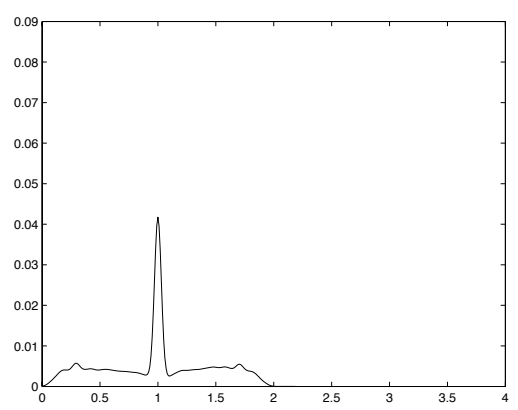

(a)

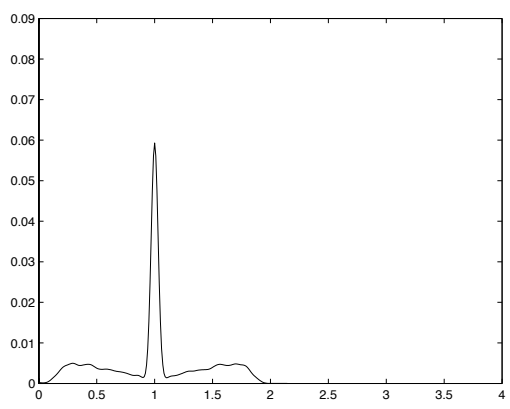

(b)

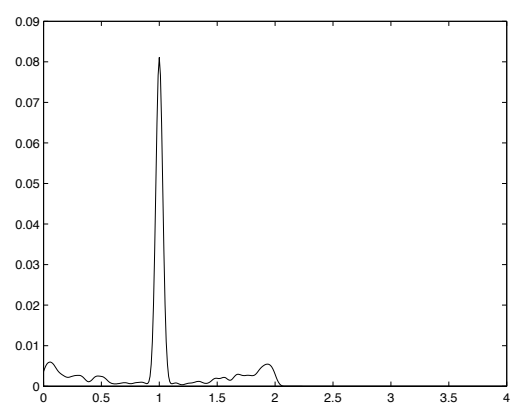

(c)

Figure 5: Protein-protein interaction networks. Data are collected from http://www.cosin.org/ [download date: 25th September, 2005]. (a) Saccharomyces cerevisiae. Size of the network is 3930. (b) Helicobacter pylori. Size of the network is 710. (c) Caenorhabditis elegans. Size of the network is 314 . 


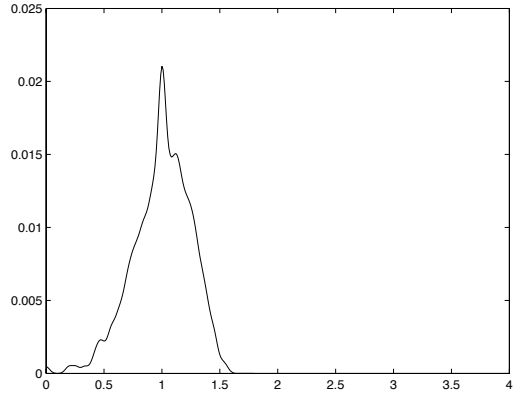

(a)

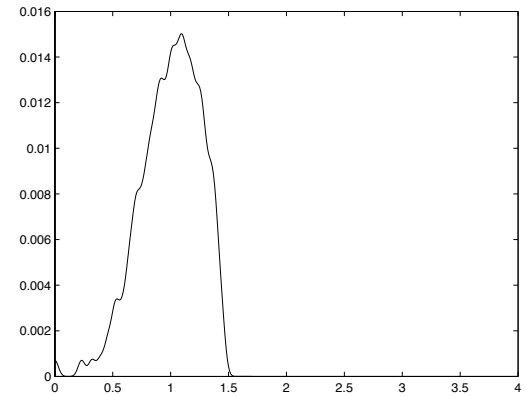

(b)

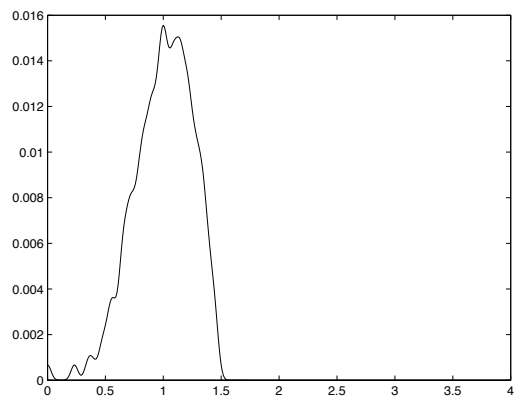

(c)

Figure 6: Neuronal connectivity. (a) Caenorhabditis elegans. Size of the network is 297. Data used in 30. 31. Data Source: http://cdg.columbia.edu/cdg/datasets/ [Download date: 18th Dec. 2006]. (b) Caenorhabditis elegans (animal JSH, L4 male) in the nerve ring and RVG regions. Network size is 190. Data source: Data is assembled by J. G. White, E. Southgate, J. N. Thomson, S. Brenner 31] and was later revisited by R. M. Durbin (Ref. http://elegans.swmed.edu/parts/ ). [Download date: 27th Sep. 2005]. (c) Caenorhabditis elegans (animal N2U, adult hermaphrodite) in the nerve ring and RVG regions. Network size is 199. Data source: Data is assembled by J. G. White, E. Southgate, J. N. Thomson, S. Brenner 31 and was later revisited by R. M. Durbin (Ref. http://elegans.swmed.edu/parts/ ). [Download date: 27th Sep. 2005]. 


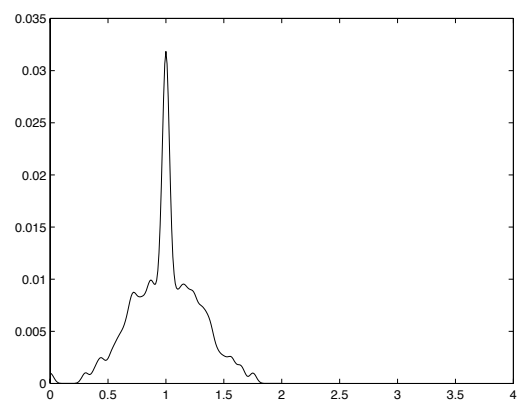

(a)

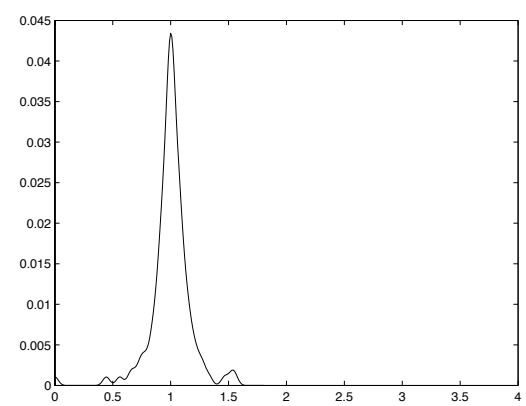

(b)

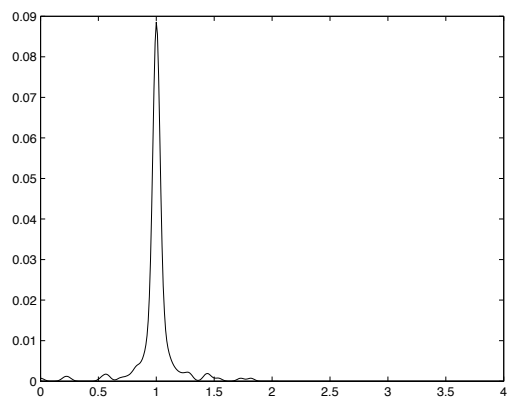

(c)

Figure 7: Food-web. (a) From "Ythan estuary". Data downloaded from http://www.cosin.org/. [Download Date 21st December, 2006]. Size of the network is 135. (b) From "Florida bay in dry season". Data downloaded from http://vlado.fmf.uni-lj.si/pub/networks/data/ (main data resource: Chesapeake Biological Laboratory. Web link: http://www.cbl.umces.edu/). [Download Date 21st December, 2006]. Size of the network is 128. (c) From "Little rock lake". Data downloaded from http://www.cosin.org/. [Download Date 21st December, 2006]. Size of the network is 183. 